\title{
Primary surgical care of cleft lip and palate patients
} Ahmad Mohammad Abdel-Moniem Aggag, Prof./ Samia Mohammed Ahmed Saied (MD), Dr/ Sherif Ahmed AbdelAal Bakri (MD)

Cleft and Craniofacial Unite, Plastic Surgery Department, Sohag University Hospital, Sohag, Egypt.

\section{Abstract:}

Social factors and gender of patients with cleft lip and palate have a major impact in the medical and surgical care yet this impact is not fully reviewed. The aim of the present study was to investigate if there is any difference between males and females in the primary surgical treatment of cleft lip and palate patients and the delayed presentation of cases with cleft lip and palate.

This is a retrospective study of 343 patients with total number of 720 primary surgical procedures, Each procedure categorized into primary lip and primary palate repair. Then the age of first surgical procedure is then analyzed and compared according to gender of patients. The result of our study show that there is no statistically significant differences in the number of primary surgeries or the age of first operation but the mean of the age of first operation is higher than our protocol for primary repair. This study give an alert about the care given to cleft patients with delayed primary repair of cleft patients.

\section{Introduction}

Cleft lip and palate (CLP) is the most common congenital craniofacial abnormality occurring in about 1:1000 live births. (1)

Cleft lip with or without cleft palate is most frequent in males but isolated cleft palate is typically more in females, the sex ratio varies with severity of the cleft, presence of additional malformations, paternal age, and possibly number of affected siblings in a family (2).

Cleft lip (CL) is defined as a congenital abnormality of the primary palate anterior to the incisive foramen. It may be complete, incomplete or microform, unilateral or bilateral, and may involve a palatal cleft (CLP). A cleft palate (CP) is a congenital abnormality of the secondary palate and may be complete or incomplete, unilateral or bilateral, or sub mucous. CLP is epidemiologically and aetiologically distinct from isolated $\mathrm{CP}$ (3).

The diagnosis of a child with a cleft places an enormous emotional burden on the child's parents and family. Interaction and bonding between the infant and mother may be impaired by feeding difficulties and feelings of maternal guilt. As the child grows, poor self-esteem and impaired socialization may affect peer relationships and produce an overdependence on adults (4).

A congenital cleft lip is a deformity that has significant physical and psychologic impact. Successful repair of the cleft lip deformity is a challenging and rewarding task. Many surgical repairs have been proposed Understanding and application of these techniques can aid the cleft surgeon in maximizing function and appearance of a child born with a cleft deformity (5).

Multidisciplinary care for cleft lip and palate (CLP) is aimed primarily at optimising the quality of speech and correcting facial disfigurement. These are only two of many aspects of cleft care that are addressed by a cleft team. Although psychological support may 
SOHAG MEDICAL JOURNAL

Vol. 22 No.3 October 2018
Primary surgical care of cleft lip and palate patients Ahmad Mohammad Abdel-Moniem Aggag

be available in high volume cleft centres, the psychological outcome of cleft care is rarely investigated and the emotional aspects of cleft rehabilitation, such as coping with teasing, are largely neglected. Self satisfaction with has an important influence upon psychological wellbeing (6).

It has been studied how gender affects health care in a number of aspects and various inequalities in different treatments are shown to exist between males and females. In development of new medicines studies are implemented mainly on men. Also studies have shown that men have better access to different treatments than women.(7).

The aim of the present study was to investigate difference between males and females in the primary surgical treatment of cleft lip and palate patients and the delayed presentation of cases with cleft lip and palate.

\section{Patient and Methods}

This is a retrospective study that was carried out on patients that had been managed in Sohag Cleft and Craniofacial Unit for CLP taking in consideration gender of cases.

We investigated 364 cleft patients ( 134 female and 230 male) of which

\section{Result}

\begin{tabular}{|c|c|c|c|c|}
\hline \multirow{2}{*}{ UCLB } & Gender & $\begin{array}{c}\text { N of } \\
\text { cases }\end{array}$ & $\begin{array}{c}\text { Total } \\
\text { pr. }\end{array}$ & $\begin{array}{c}\text { Primary } \\
\text { pr. }\end{array}$ \\
\hline & Male & 126 & 319 & 255 \\
\cline { 2 - 5 } & Female & 82 & 240 & 174 \\
\cline { 2 - 5 } & Total & 208 & 559 & 429 \\
\hline \multirow{3}{*}{ BCLB } & Male & 93 & 245 & 194 \\
\cline { 2 - 5 } & Female & 42 & 134 & 97 \\
\cline { 2 - 5 } & Total & 135 & 379 & 291 \\
\hline
\end{tabular}

Table (1): Number of cases with UCLP and BCLP

Cases with unilateral complete cleft lip and palate (UCLP):

There was no statistically significant difference in the numbers of primary surgical procedures between males, $2.02 \pm 0.05$ (mean \pm SEM), and females, $2.12 \pm 0.068[\mathrm{p}=$ $0.242]$. 
SOHAG MEDICAL JOURNAL Vol. 22 No.3 October 2018
Primary surgical care of cleft lip and palate patients Ahmad Mohammad Abdel-Moniem Aggag

There was no statistically significant difference in the age of the first surgical procedure between males, $7.7 \pm 1$ (mean \pm SEM), and females, $10.9 \pm 3.2[\mathrm{P}=0.275$ ].

Cases with bilateral complete cleft lip and palate (BCLP).

No statistically significant difference in the numbers of primary surgical procedures between males, $2.09 \pm 0.066$ (mean \pm SEM), and females, $2.3 \pm 0.1[\mathrm{p}=$ $0.067]$.

There was no statistically significant difference in the age of the first surgical procedure between males, $10.4 \pm 2.6$ (mean \pm SEM), and females, $8.99 \pm 2.5[\mathrm{P}=$ $0.735]$.

\begin{tabular}{|c|c|c|c|c|c|c|c|}
\hline \multirow{5}{*}{ 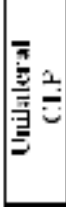 } & & Giender & $\mathrm{N}$ & Mran & S.T) & S.F..M & P-yalus \\
\hline & \multirow{4}{*}{$\begin{array}{l}\text { Primary } \\
\text { prosedire } \\
\text { Age nf } \\
\text { tust } \\
\text { prosediue }\end{array}$} & malc & 126 & 2.0 & .57 & .05 & \multirow[t]{3}{*}{.212} \\
\hline & & \multirow{2}{*}{$\begin{array}{l}\text { feruule } \\
\text { malc: }\end{array}$} & 82 & 2.1 & .62 & .07 & \\
\hline & & & 126 & 77 & 122 & 108 & \\
\hline & & \multicolumn{2}{|c|}{ ferayle 82} & 10.9 & $2 i_{0} 0$ & 3.20 & \\
\hline \multirow{4}{*}{ 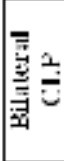 } & \multirow{4}{*}{$\begin{array}{l}\text { Primary } \\
\text { prosedine } \\
\text { Mge of } \\
\text { irst } \\
\text { prozedire }\end{array}$} & male & 93 & 2.08 & .6 .37 & .066 & .067 \\
\hline & & feruyle & 42 & 2.30 & .68 & .105 & \multirow{3}{*}{.735} \\
\hline & & malc & 93 & 10.4 & 25.3 & 2.6 & \\
\hline & & finalc & 42 & 8.98 & 16.4 & 2.5 & \\
\hline
\end{tabular}

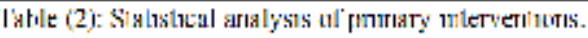

\section{Discussion}

Many gender differences in care of patients with surgical and medical conditions have been recognized, with great impact of sex and gender in treatment of patients and huge importance of gender for understanding health and illness. Unfortunately, gender effect on patients care largely neglected. Data on differences of medical practice does not sufficiently take gender into account in diagnosis, treatment or management of patients.

the framework which links gender to the social, economic and biological determinants and consequences of diseases is applicable to other medical conditions too including cleft patients.

The aim of the present study was to investigate if there is any difference in the primary surgical treatment between males and females with cleft lip and palate

As regard health care males receives more medical treatments than females because of social, economic and biological factors, also focusing on the male model rather than a female one to compare a medical or surgical methods of treatments increasing the gape of care between both(8)

As the same protocol for surgical care in our unite was applied to all cases whether male or female without any differentiation or discrimination, the statistical analysis of primary surgical care of cleft patients according to their sex found that the primary surgical care was nearly equal with statistically no significant difference in number of primary surgical occasions .

The age of first operation done for primary correction of the lip and palate deformity was analyzed for both males and females ,first the mean of operations calculated then statistical comparison done between both means, showing that there was no significant difference in the age of the first surgical procedure between males and females as both came for surgical correction nearly at the same age, but the mean of the age of the first operation found to be higher than our protocol which is 3 months for lip repair.

In BCLP mean of age of first operation was 10.4 months in males 
SOHAG MEDICAL JOURNAL Vol. 22 No.3 October 2018

and 8.9 months for females, Also in UCLP mean of age of first operation was 7.7 months in males and 10.9 months for females.

To explain this high mean of age of first operation close search in the range of age for primary repair done finding that un neglectable number of cases had postponed their first operation with range of (335 months for females and 431 months for males), this late presentation of some cases resulting in higher mean than our designed protocol, This late presentation can be explained by economical, religious ,educational factors for those cases.

The suggested causes for this late presentations include the expensive cost of operations, the cost of post operative care and treatments, parents think that his child may not with stand operations at this young age and prefer to postpone any surgical correction till patient reach 2 years and some time school age or even more specially with minor deformities as incomplete lip, Also this late presentation found in males and females suggesting that it's not related to gender discrimination.

In conclusion the evaluation of primary care of patients with cleft lip and palate show no statistically significant difference between males and females but the delayed presentation of cases and its effect on delayed care give an alert about societal awareness of cleft care and importance of early repair.
Primary surgical care of cleft lip and palate patients Ahmad Mohammad Abdel-Moniem Aggag

\section{Referances}

1- F.-J. Kramer, C. Baethge, B. Sinikovic, H. Schliephake: An analysis of quality of life in 130 families having small children with cleft lip/palate using the impact on family scale. Int. J. Oral Maxillofac. Surg. 2007; 36: 1146-1152

2- Peter A Mossey, Julian Little, Ron G Munger, Mike J Dixon, William C Shaw Cleft lip and palate The Lancet, Volume 374, Issue 9703, 21-27 November 2009, Pages 1773-1785

3- Tim Goodacre, , Marc C. Swan, Cleft lip and palate: current management Paediatrics and Child Health, Volume 18, Issue 6, June 2008, Pages 283-292

4- Joanna C. Mitchell, Robert J. Wood Management of cleft lip and palate in primary care, Journal of Pediatric Health Care Volume 14, Issue 1, January-February 2000, Pages 13-19

5-Jonathan M. Sykes, Travis T. Tollefson, Management of the cleft lip deformity Facial Plastic Surgery Clinics of North America Volume 13, Issue 1, February 2005, Pages 157-167

6-S.R. Turner, P.W.N. Thomas, T. Dowell, N. Rumsey, J.R. Sandy Psychological outcomes amongst cleft patients and their families British Journal of Plastic Surgery, Volume 50, Issue 1, 1997, pp. 1-9

7- Linn S.K. a comparison of surgical treatment between male and female patients with complete unilateral cleft lip and palate university of Gothenburg 2013 p 8

8- Vlassoff C. 2007. Gender differences in determinants and consequences of health and illness. Journal of health, population, and nutrition, 25, 47 
SOHAG MEDICAL JOURNAL

Vol. 22 No.3 October 2018
Primary surgical care of cleft lip and palate patients Ahmad Mohammad Abdel-Moniem Aggag 